\title{
Educating the Next Generation of Physicians in Interprofessional Collaboration
}

\author{
Leslie Sheu, MD, Ryan Laponis, MD, MSci, and Katherine Julian, MD
}

Division of General Internal Medicine, University of California, San Francisco, CA, USA.

KEY WORDS: interprofessional collaboration; interprofessional education; medical education.

J Gen Intern Med 31(8):823-5

DOI: $10.1007 /$ s11606-016-3731-1

(c) Society of General Internal Medicine 2016

E ffective interprofessional collaboration (IPC) is essential for providing safe, efficient, and comprehensive patient care. ${ }^{1}$ This is particularly true in light of the increased prevalence and complexity of chronic disease, increased attention on improving access to care, and greater focus on patient safety and quality. In 2001, the Institute of Medicine stated that interprofessional team-based care would allow healthcare professionals to address these increasingly complex needs. ${ }^{2}$ Since then, we have seen reports that one doctor can no longer "do it all," and that a team-based approach is central to a highperforming practice. ${ }^{3}$ We have seen the successful implementation of such models through patient-centered medical homes and accountable care organizations, and as physicians, we understand the critical importance of our interprofessional colleagues, in both inpatient and outpatient settings, for providing and coordinating care for our patients.

In 2009, six national associations of schools across health professions developed an expert consensus on core competencies in IPC to encourage and promote interprofessional education. ${ }^{4}$ Since then, medical educators at all levels of training have begun to emphasize IPC as a necessary skill. The internal medicine residency reporting milestones include respectful interprofessional interaction among healthcare providers as a professionalism milestone and effective interprofessional communication as an interpersonal communication milestone. ${ }^{5}$ Resident trainees must demonstrate competence in these areas prior to completion of residency. However, ways of identifying curricular gaps in order to determine the most effective methods of teaching IPC have not been clearly defined.

In this issue of JGIM, Zabar and colleagues report on results from a single internal medicine residency program assessing IPC in residents completing observed structured clinical exams (OSCEs). ${ }^{6}$ Using performance-based assessment in the OSCEs, the authors developed several checklist items for

Published online May 5, 2016 standardized nurses (SN) on three interprofessional standardized patient (SP) encounters. IPC skills were at least partially achieved by the majority of residents, but fewer than half of residents completed most items in a "well-done" manner, and standard deviations were high. Zabar and colleagues also found that the specific case simulation influenced IPC scores, with residents receiving more scores of "well done" on a case of chest pain, and fewer such scores on a case of diabetes management. IPC performance was not significantly correlated with any of the core clinical skills (such as patient communication, history taking, or physical exam) assessed in the OSCE. Qualitative analysis on written SN comments revealed two primary patterns observed: (1) either the resident effectively interacted with the SP but not the SN, or (2) the resident effectively interacted with both, but had difficulty handling the core challenges of the case. Residents agreed that further information and clarification around interprofessional roles of team members would be helpful, as would more purposively structured opportunities for IPC during the workday, such as huddles or round table meetings.

Zabar and colleagues' work brings up several points for further research and discussion. First, it is interesting to note that in their study, IPC skills varied based on the case. This variability suggests that it may be easier to effectively communicate with interprofessional colleagues in acute or "singleproblem" scenarios, such as that of chest pain, and more challenging in cases with increased medical complexity, such as that of diabetes management. Arguably, IPC skills are critical in both of these scenarios. Perhaps, as medical and logistical complexity increases, as in diabetes management, the prioritization of medical issues and patient counseling occurs at the expense of IPC. It is also possible that a simulated OSCE experience may not accurately represent real-life interprofessional communication that occurs in clinical practice.

Second, along similar lines, it is interesting that the authors found that skills in communicating with patients and with nurses were not correlated, and in fact, residents sometimes interacted effectively with the patient at the expense of interaction with their interprofessional colleagues. This has two implications: (1) communicating with patients and communicating with nurses are truly unique skills and must be addressed differently, starting in medical school, and (2) physicians may need better training in order to communicate equally well with nurses and patients during a patient encounter. 
Third, the residents' self-awareness of their IPC skills and recommendations for further training were notable. Roles and responsibilities of team members are complex, and anecdotally vary significantly from one clinical setting to the next. Thus, clear mutual understanding of roles is a key first step to achieving competence in IPC. One way to achieve this might be through huddles; perhaps less impactful ways include site-specific training sessions or written documents. Built-in workplace-based structured IPC activities and face time with interprofessional colleagues are practical ways of increasing and improving IPC, rather than carving out additional structured curricular interventions. Increased opportunities for face-to-face IPC not only can enhance understanding of mutual roles, but can also foster the development of individual relationships and mutual understanding and respect. Indeed, high-functioning primary care practices often improve team communication through co-location, scheduled huddles, and regular team meetings. ${ }^{7}$ In a 2009 Cochrane review, practicebased IPC interventions were shown to improve both processes and outcomes in healthcare, though further studies are needed to understand generalizability. ${ }^{8}$ Perhaps these experiential learning opportunities in authentic clinical settings - with deliberate, targeted goals based on identified gaps - may be the best way to teach IPC to physicians in training.

Finally, Zabar and colleagues encourage us to think about how we design future IPC curricular interventions, and provide us with a tool to assess these interventions through structured OSCEs and IPC-specific assessment questions. There are a few limitations to the feasibility and generalizability of their model. First, at the authors' institution, a resident OSCE was already part of their resident assessment— perhaps making it easier to adjust specific cases for an IPC focus. OSCEs are not universal among residencies, and require substantial resources for case development and SP/SN training - as well as residency support in setting aside time for these assessments, in light of already compressed time in the workplace due to duty hours. Second, while the internal validity of questions is strong, there is significant variability among the three cases. This study is limited to residents at a single institution, so it is unclear how this assessment tool may translate to other programs. However, the OSCE holds exciting potential as a tool for assessing residents' interprofessional skills as required for the reporting milestones. Programs that do have the time, financial and faculty resources, and programmatic infrastructure for further IPC skill development, such as simulation sessions, can assess the benefit of their curricula by tracking scores before and after their intervention. Medical schools, which are more likely to have existing OSCEs, could similarly test this assessment tool on their student cohort. Furthermore, with ongoing robust evaluation of the validity of this tool, IPC skills could be tracked over time throughout medical training.
The study by Zabar and colleague highlights the fact that medical educators are likely not providing internal medicine residents with adequate training on IPC skills. As we continue to strive for improved IPC training, this study brings to bear three important points:

1. "We can't improve what we don't measure." This study helps us begin to understand where the gaps are in resident skills with regard to interaction with nurses, and might guide us in targeting specific areas for curriculum development efforts. Additionally, cultivation of these skills in real life requires iterative feedback; thus an OSCE model for assessment makes sense conceptually, as feedback is a key component of this model.

2. "Junk in = junk out." In other words, how we measure these skills is important, because if we don't get the measurement right, we will be teaching to the wrong skill set. This article provides the generation of a behavioral-anchored checklist for IPC competencies, and further use and validation of this tool will be important to see if it might be used more broadly.

3. "IPC is a two-way (or multi-way) street." Ultimately, all team members should be trained and authentically assessed in IPC skills. Truly interprofessional curricula or simulations to simultaneously train our next generation of physicians alongside nurses, pharmacists, and other healthcare professional colleagues are critical for developing a mutual understanding of roles and responsibilities and fostering mutual respect. This would make for true interprofessional education.

The current dialogue and efforts on the part of many with regard to the study and assessment of IPC is exciting and greatly needed. We look forward to future innovations in both curricular development and IPC skills assessment.

Corresponding Author: Leslie Sheu, MD; Division of General Internal MedicineUniversity of California, San Francisco, 1545 Divisadero Street, San Francisco, CA 94115, USA (e-mail: leslie.sheu@ucsf.edu).

\section{Compliance with ethical standards:}

Conflict of interest: The authors have no conflicts of interest to report.

\section{REFERENCES}

1. Craig C, Eby D, Whittington J. Care Coordination Model: Better Care at Lower Cost for People with Multiple Health and Social Needs. IHI Innovation Series white paper. Cambridge: Institute for Healthcare Improvement; 2011.

2. Institute of Medicine Committee on Quality of Health Care in America. Crossing the quality chasm: a new health system for the 21 st century. Washington: National Academy Press; 2001.

3. Bodenheimer T, Ghorob A, Willard-Grace R, Grumbach K. The 10 building blocks of high-performing primary care. Ann Fam Med. 2014;12(2): 166-171.

4. Schmitt M, Blue A, Aschenbrener CA, Viggiano TR. Core competencies for interprofessional collaborative practice: reforming health care by transforming health professionals' education. Acad Med. 2011;86(11):1351. 
5. Green ML, Aagaard EM, Caverzagie $\mathbf{K J}$, et al. Charting the road to competence: developmental milestones for internal medicine residency training. J Grad Med Educ. 2009; 1(1):5-20.

6. Zabar S, Adams J, Kurland S, et al. Charting a key competency domain: understanding resident physician interprofessional collaboration (IPC) skills. J Gen Int Med. doi:10.1007/s11606-016-3690-6.
7. Sinsky CA, Willard-Grace R, Schutzbank AM, Sinsky TA, Margolius D, Bodenheimer T. In search of joy in practice: a report of 23 high-functioning primary care practices. Ann Fam Med. 2013;11(3):272-278.

8. Zwarenstein $\mathbf{M}$, Goldman $\mathbf{J}$, Reeves $\mathbf{S}$. Interprofessional collaboration: effects of practice-based interventions on professional practice and healthcare outcomes. Cochrane Database Syst Rev. 2009;3, CD000072. 